\title{
Rare frequency of gene variation and survival analysis in thymic epithelial tumors
}

This article was published in the following Dove Press journal:

OncoTargets and Therapy

14 October 2016

Number of times this article has been viewed

\author{
Zhengbo Song ${ }^{1,2, *}$ \\ Xinmin $\mathrm{Yu}^{1,2, *}$ \\ Yiping Zhang ${ }^{1,2}$ \\ 'Department of Medical Oncology, \\ Zhejiang Cancer Hospital, ${ }^{2}$ Key \\ Laboratory Diagnosis and Treatment \\ Technology on Thoracic Oncology, \\ Zhejiang Province, Hangzhou, People's \\ Republic of China \\ *These authors contributed equally \\ to this work
}

Correspondence: Zhengbo Song

Department of Medical Oncology,

Zhejiang Cancer Hospital, 38

Guangji Road, Hangzhou 310022,

People's Republic of China

Tel $+8657|88| 22082$

Fax +86 57I 88I2 2088

Email songzhengbo83@163.com
Objective: Thymic epithelial tumor (TET) is a rare mediastinal neoplasm and little is known about its genetic variability and prognostic factors. This study investigated the genetic variability and prognostic factors of TET.

Patients and methods: We sequenced 22 cancer-related hotspot genes in TET tissues and matched normal tissues using Ampliseq Ion Torrent next-generation technology. Overall survival was evaluated using Kaplan-Meier methods and compared with log-rank tests.

Results: A histological analysis of 52 patients with a median age of 52 years showed 15 patients (28.8\%) with thymic carcinoma, five with type A thymoma (9.6\%), eight with type AB (15.4\%), six with type B1 (11.5\%), nine with type B2 (17.3\%), and nine with type B3 thymoma (17.3\%). Three gene mutations were identified, including two with PIK3CA mutation and one with $E G F R$ mutation. The three patients with mutant genes included two cases of thymoma (one with $E G F R$ and the other with PIK3CA mutation) in addition to a case of thymic carcinoma (PIK3CA mutation). The 5-year survival rates were $77.7 \%$ in all patients. The 5-year survival rates were $93.3 \%, 90.0 \%, 76.9 \%$, and $22.9 \%$ corresponding to Masaoka stages I, II, III, and IV $(P<0.001)$. The 5-year survival rates were $100 \%, 100 \%, 83.3 \%, 88.9 \%, 65.6 \%$, and $60.9 \%$ in the histological subtypes of A, AB, B1, B2, and B3 thymomas, and thymic carcinoma, respectively $(P=0.012)$.

Conclusion: Hotspot gene mutations are rare in TET. PIK3CA and EGFR mutations represent candidate driver genes and treatment targets in TET. Masaoka stage and histological subtypes predict the survival of TET.

Keywords: thymic epithelial tumors, gene mutation, prognosis

\section{Introduction}

Thymic epithelial tumor (TET) is a relatively uncommon tumor of thymic epithelial origin. ${ }^{1}$ Previous studies demonstrated that radical surgery is the only effective intervention for long-term survival. ${ }^{2,3}$ However, local recurrence or distant metastasis may occur in some patients even after complete resection. There is no effective treatment for advanced and recurrent disease and the prognosis is poor. ${ }^{4}$

Several studies with limited number of patients have shown that targeted treatment may be effective in specific cases. ${ }^{5-7}$ Partial efforts have been made to identify the driver genes of TET in order to develop treatment strategies. ${ }^{8,9}$ Most of the studies focus on single driver genes, such as EGFR, HER2, Kit, and KRAS. ${ }^{10-14}$ Few studies evaluated the gene status in TET with high-throughput technology. ${ }^{15}$

Several studies have demonstrated that Masaoka stage was an important prognostic factor for TET. However, the role of other factors, including histological subtypes and myasthenia gravis, remains controversial. ${ }^{16-17}$ 
In the present study, we identified mutations associated with tumorigenesis using next-generation sequencing (NGS) of 22 hotspot genes in a series of 52 TET patients. Furthermore, we evaluated the survival of 52 TET patients to investigate the prognostic factors.

\section{Patients and methods}

\section{Patients}

Fifty-two patients with pathologically confirmed TET, who underwent diagnosis between January 2007 and December 2012, were identified in Zhejiang Cancer Hospital. Two pathologists blinded to patients' clinical pathology reviewed the samples. The patients were clinically staged according to the Masaoka-Koga system. ${ }^{18}$ The histologic types were based on the 2004 World Health Organization classification. ${ }^{19}$ The histologic subtypes included type A, AB, B1, B2, B3, and thymic carcinomas. The study was approved by the Ethics Committee of Zhejiang Cancer Hospital and all patients provided written informed consent for biomarker analysis and to have their data used in this study.

\section{NGS detection methods}

Microscopy was used to ensure that the tumor tissues analyzed had more than $20 \%$ tumor contents. Tumor DNA was isolated from formalin-fixed and paraffin-embedded sections using the QIAamp DNA Mini kit (Qiagen GmbH, Hilden, Germany), following manufacturer's instructions. Ten nanograms of DNA were used to prepare the bar-coded libraries with the Ion AmpliSeq ${ }^{\mathrm{TM}}$ Library kit 2.0 (Thermo Fisher Scientific, Waltham, MA, USA). The panel was used to analyze 1,800 mutational hotspots and targeted regions in 22 genes: $E G F R, K R A S, B R A F$, PIK3CA, AKT1, ERBB2, PTEN, NRAS, STK11, MAP2K1, ALK, DDR2, CTNNB1, MET, TP53, SMAD4, FBXW7, FGFR3, NOTCH1, ERBB4, FGFR1, and FGFR2. Template preparation and enrichment were performed with the Ion OneTouch ${ }^{\mathrm{TM}}$ two System (Thermo Fisher Scientific). Finally, sequencing was carried out using Ion 316TM chips on the ion personal genome machine (Ion PGM) System (PGM ${ }^{\mathrm{TM}}$, Thermo Fisher Scientific) and with the Ion PGM ${ }^{\text {TM }}$ Sequencing 200 kit v2. Alignment to the hg19 human reference genome and variant calling were performed by the Torrent Suite Software version 4.0.2 (Thermo Fisher Scientific). Alignments were visually checked with the Integrative Genomics Viewer (IGV) version 2.3.34..$^{20,21}$ DNA from normal tissue from all samples was also sequenced to check the germline status of the variants.

\section{Statistical analysis}

Survival curves were calculated using the Kaplan-Meier method from the start of diagnosis until death or last follow-up. Statistical analysis was performed using the SPSS 18 software (SPSS Inc., Chicago, IL, USA). $P<0.05$ was considered statistically significant. The median follow-up period was 65 months (36-118 months) and the last follow-up time was March 31, 2015.

\section{Results \\ Clinical presentation}

Totally, 52 patients, including 20 males and 32 females, with pathologically confirmed TET were enrolled. The clinicopathological characteristics are listed in Table 1. The median age at diagnosis was 52 years (range, 18-71 years). Eleven patients were current or ever smokers and 41 neversmokers. Histologically, there were five patients with type A thymoma (9.6\%) (Figure 1A), eight with type AB (15.4\%) (Figure 1B), six with type B1 (11.5\%) (Figure 1C), nine with type B2 (17.3\%) (Figure 1D), nine with type B3 thymoma (17.3\%) (Figure 1E), and 15 with thymic carcinoma (28.8\%) (Figure 1F). Pathologic stages at diagnosis included: 18 patients with stage I, eleven patients with stage II, 13 patients with stage III, and ten patients with stage IV disease. Totally, four patients were identified as having extrathymic malignancies in addition to thymoma (three patients) and thymic carcinoma (one patient). The incidence of extrathymic malignancies in patients with thymoma was $8.1 \%$ for thymoma and $6.7 \%$ for thymic carcinoma. The four extrathymic malignancies included lung cancer $(n=1)$,

Table I Clinical characteristics of the study population

\begin{tabular}{ll}
\hline & Number \\
\hline Sex & 20 \\
Male & 32 \\
Female & \\
Age (years) & 52 \\
Median & $18-71$ \\
Range & 21 \\
$<50$ & 31 \\
$\geq 50$ & \\
Smoking status & 41 \\
Never & 11 \\
Former/current & \\
Histology & 5 \\
Type A & 8 \\
Type AB & 6 \\
Type BI & 9 \\
Type B2 & 9 \\
Type B3 & 15 \\
Type C & \\
Masaoka stage at diagnosis & 18 \\
I & 11 \\
II & 13 \\
III & 10 \\
IV &
\end{tabular}

Note: aHistology type is thymoma or thymic carcinoma. 

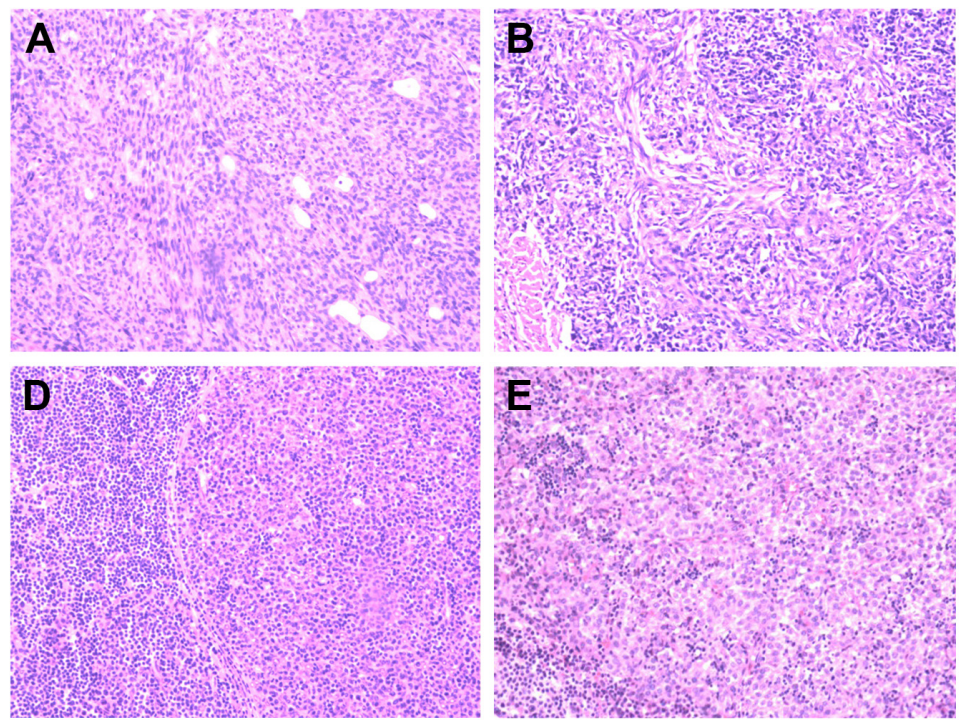
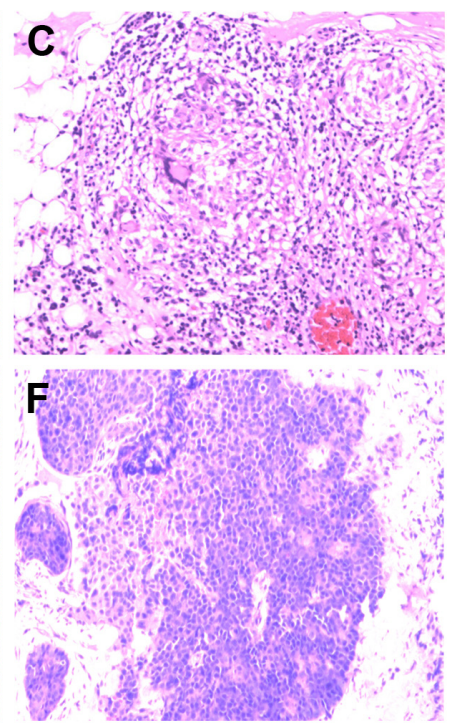

Figure I $\mathrm{H}$ and $\mathrm{E}$ stained images of the different subtypes of thymic epithelial tumors $(\times 40)$.

Notes: (A) Type A, (B) type AB, (C) type BI, (D) type B2, (E) type B3, (F) type C.

Abbreviation: $\mathrm{H}$ and $\mathrm{E}$, hematoxylin and eosin.

hepatocellular carcinoma $(\mathrm{n}=1)$, breast carcinoma $(\mathrm{n}=1)$, and colon cancer $(\mathrm{n}=1)$.

\section{Molecular analyses}

NGS analyses were performed on all the 52 cases. One patient carried an EGFR mutation (E746_750de), and two cases harbored PIK3CA mutation (both E545Q) (Table 2). No molecular aberrations were found in the other 49 patients.

\section{Treatment}

Forty-six patients underwent surgical resection, including 40 with complete resection and six with incomplete resection. The surgical approach included sternotomy $(n=25)$, thoracotomy $(\mathrm{n}=14)$, and video-assisted thoracoscopic surgery $(n=7)$. Fifteen patients were treated with radiation therapy (dose range: 40-60 Gy). Twenty-three patients received chemotherapy (six with only chemotherapy, 17 with radiotherapy and chemotherapy).

\section{Survival analyses}

The disease-free survival for the 46 patients with surgery was $57.8 \%$. Twenty-one patients received first-line chemotherapy with median progression-free survival of 8.2 months (95\% confidence interval [CI]: 5.1-10.3).

The 5-year survival rate was $77.7 \%$ in all patients. The 5-year survival rates in the subtypes A, AB, B1, B2, and B3 thymomas, and thymic carcinoma were $100 \%, 100 \%, 83.3 \%$, $88.9 \%, 65.6 \%$, and $60.9 \%$, respectively ( $P=0.012$; Figure 2$)$. The 5 -year survival rates were $93.3 \%, 90.0 \%, 76.9 \%$, and $22.9 \%$ by Masaoka stage I, stage II, stage III, and stage IV $(P<0.001$; Figure 3$)$, respectively.

No significant effects of age $(P=0.739)$, sex $(P=0.226)$, or myasthenia gravis $(P=0.832)$ on overall survival were observed. Additionally, no survival difference was found between patients with and without gene mutation ( $P=0.352$; Figure 4$)$.

\section{Discussion}

Our study showed that only three samples with two mutation types were found in 52 TET patients. Two cases of PIK3CA mutation and one with EGFR mutation were identified. To the best of our knowledge, our study represents the largest number of patients with NGS detection in TET. In addition, the Masaoka stage and histological subtypes were confirmed as prognostic factors in our cohort.

Table 2 Clinical characteristics in gene alteration patients

\begin{tabular}{lllllll}
\hline Case & Sex/age (years) & Smoking history & Histology $^{\mathbf{a}}$ & Gene type & Treatment & OS/months \\
\hline I & Female/48 & Yes & B2 & PIK3CA & Surgery & $85+$ \\
2 & Male/55 & No & C & PIK3CA & Surgery + radiotherapy & 46 \\
3 & Female/46 & No & B3 & EGFR & Surgery & 45 \\
\hline
\end{tabular}

Note: aHistology type is thymoma or thymic carcinoma.

Abbreviation: OS; overall survival. 


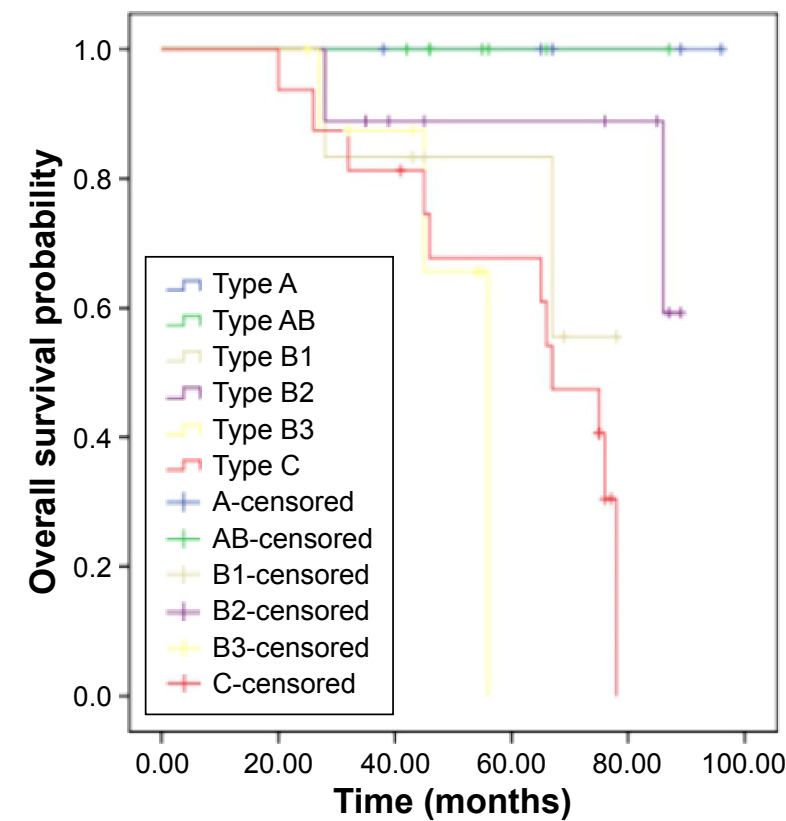

Figure 2 Kaplan-Meier curve of overall survival of different histological subtypes $(P=0.012)$.

Thymomas are defined as neoplasms arising from or exhibiting differentiation toward thymic epithelial cells, usually with a variable component of nonneoplastic lymphocytes. Thymic carcinomas are malignant epithelial tumors with overt cytologic atypia, almost invariably invasiveness and lack the

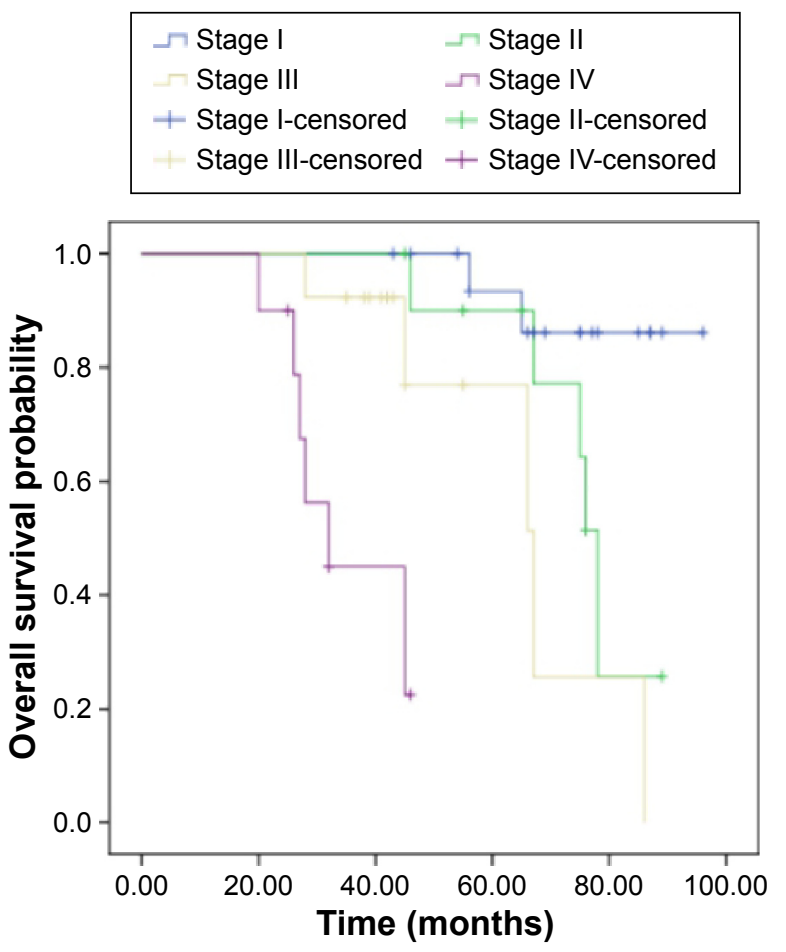

Figure 3 Kaplan-Meier curve of overall survival of different Masaoka stage $(P<0.001)$.

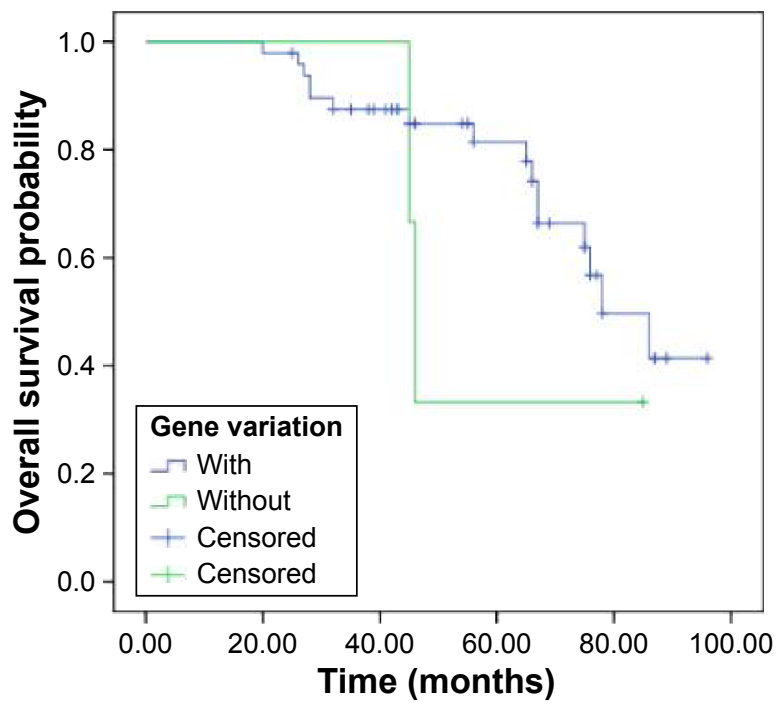

Figure 4 Kaplan-Meier curve of overall survival in patients with $(n=3)$ and without $(n=49)$ gene variation $(P=0.352)$.

organotypic features of thymomas. From a histological point of view, some gene variations commonly seen in epithelial origin tumors may be found in TET. PIK3CA pathways play an important role in carcinoma proliferation and metastasis. The frequency of PIK3CA mutations is $\sim 2 \%-5 \%$ in other solid carcinomas..$^{22,23}$ Data pertaining to PIK3CA mutations in thymoma and thymic carcinoma have not been widely reported. Until now, only one study by Wheler et al reported a PIK3CA mutation in one of the 12 TET patients. ${ }^{24}$ In the present cohort, two PIK3CA mutations were detected in thymoma with a frequency of $3.8 \%$. Epidermal growth factor receptor (EGFR) mutations have been identified as driver genes in non-small-cell lung cancer (NSCLC) at a frequency of $40 \%-50 \%$ in the East Asian population. The EGFR mutations in TET have been identified in previous studies. ${ }^{25,26}$ No representative clinical characteristics associated with EGFR mutations were found, although most patients were diagnosed with thymoma but not thymic carcinoma. In the current study, our patient who carried the EGFR mutation represented type $\mathrm{B} 3$.

No targeted drugs are approved in TET due to its rarity and lack of explicit molecular targets. Patients with advanced/ metastatic thymoma or thymic carcinoma benefited from mammalian target of rapamycin inhibitor-based therapy in a Phase I study with a median time to treatment failure of 11.6 months. ${ }^{24}$ However, no PIK3CA mutation was found in patients responding to $m T O R$ inhibitor treatment. Higher serum concentrations of $V E G F, b-F G F$, and $c K I T$ expression were observed in patients with thymic carcinoma. One Phase II trial investigated the activity of sunitinib, an 
oral tyrosine kinase inhibitor, including $V E G F R, K I T$, and $P D G F R$ in TET patients. ${ }^{27}$ The results showed that sunitinib showed clinical activity in heavily pretreated patients with thymic carcinoma. EGFR-tyrosine kinase inhibitors (TKIs) displayed adequate efficacy in NSCLC patients who carried EGFR mutations. A Phase II study of gefitinib for advanced thymic malignancies showed that disease was controlled in 15 patients, including partial response in one case and stable disease in 14 others. ${ }^{25}$ However, no association was found between EGFR mutations and gefitinib efficacy. The role of EGFR mutations in carcinomas may be different in thymoma compared with NSCLC. In our cohort, only one patient with thymoma carried the EGFR mutation. Unfortunately, no EGFR-TKI was administered to this patient, and the efficacy of EGFR-TKI is unknown.

The prognostic role of clinical and pathologic factors remains controversial in TET. ${ }^{2-4,16-17}$ The Masaoka pathological stage represents an important prognostic factor for thymic carcinoma. In the current study, the Masaoka pathological stage was associated with significant effects on the prognosis of thymoma and thymic carcinoma. In addition, we found that histological subtypes hold the key to survival in patients with TET. However, other clinical factors, such as sex, age, and myasthenia gravis, played no role in survival.

The occurrence of extrathymic malignancy in TET patients has not been delineated in the published studies, with an incidence of $\sim 10 \%$ having been reported. ${ }^{28,29}$ Four of 52 patients were found with extrathymic malignancy in the present study, which is consistent with previous studies.

\section{Limitations}

The major limitation of this study was the relatively small sample size to evaluate gene status and survival. Therefore, a larger series of patients and high-throughput sequencing with additional genes are needed to identify the prevalence of mutations and survival in TET. Furthermore, functional analyses of the mutations are needed to determine the molecular mechanisms of tumorigenesis.

\section{Conclusion}

In conclusion, this study reports the incidence of $P I K 3 C A$ and EGFR mutations in some TET patients. These mutations may represent promising therapeutic candidates. In addition to Masaoka stage, histological subtypes predict the survival of patients with TET.

\section{Disclosure}

The authors report no conflicts of interest in this work.

\section{References}

1. Engels EA, Pfeiffer RM. Malignant thymoma in the United States: demographic patterns in incidence and associations with subsequent malignancies. Int J Cancer. 2003;105(4):546-551.

2. Zhu L, Zhang J, Marx A, Weiss C, Fang WT. Clinicopathological analysis of 241 thymic epithelial tumors-experience in the Shanghai Chest Hospital from 1997-2004. J Thorac Dis. 2016;8(4):718-726.

3. Fang W, Fu J, Shen Y, et al. Management of thymic tumors-consensus based on the Chinese Alliance for Research in Thymomas Multiinstitutional retrospective studies. J Thorac Dis. 2016;8(4):641-645.

4. Hishida T, Nomura S, Yano M, et al. Long-term outcome and prognostic factors of surgically treated thymic carcinoma: results of 306 cases from a Japanese Nationwide Database Study. Eur J Cardiothorac Surg. 2016;49(3):835-841.

5. Bisagni G, Rossi G, Cavazza A, Sartori A, Gardini G, Boni C. Long lasting response to the multikinase inhibitor Bay 43-9006 (sorafenib) in a heavily pretreated metastatic thymic carcinoma. J Thorac Oncol. 2009;4(6):773-775.

6. Strobel P, Bargou R, Wolff A, et al. Sunitinib in metastatic thymic carcinomas: laboratory findings and initial clinical experience. Br J Cancer. 2010;103(2):196-200.

7. Rajan A, Carter CA, Berman A, et al. Cixutumumab for patients with recurrent or refractory advanced thymic epithelial tumours: a multicentre, open-label, phase 2 trial. Lancet Oncol. 2014;15(2):191-200.

8. Petrini I, Meltzer PS, Kim IK, et al. A specific missense mutation in GTF2I occurs at high frequency in thymic epithelial tumors. Nat Genet. 2014;46(8):844-849.

9. Zettl A, Strobel P, Wagner K, et al. Recurrent genetic aberrations in thymoma and thymic carcinoma. Am J Pathol. 2000;157(1):257-266.

10. Yoh K, Nishiwaki Y, Ishii G, et al. Mutational status of EGFR and KIT in thymoma and thymic carcinoma. Lung Cancer. 2008;62(3): 316-320.

11. Girard N, Shen R, Guo T, et al. Comprehensive genomic analysis reveals clinically relevant molecular distinctions between thymic carcinomas and thymomas. Clin Cancer Res. 2009;15(22):6790-6799.

12. Pan CC, Chen PC, Wang LS, Lee JY, Chiang H. Expression of apoptosis-related markers and HER-2/neu in thymic epithelial tumours. Histopathology. 2003;43(2):165-172.

13. Strobel P, Hartmann M, Jakob A, Mikesch K, et al. Thymic carcinoma with overexpression of mutated KIT and the response to imatinib. New Engl J Med. 2004;350(25):2625-2626.

14. Sasaki H, Yano M, Fujii Y. Evaluation of Kras gene mutation and copy number in thymic carcinomas and thymomas. $J$ Thorac Oncol. 2010;5(10):1715-1716.

15. Shitara M, Okuda K, Suzuki A, et al. Genetic profiling of thymic carcinoma using targeted next-generation sequencing. Lung Cancer. 2014;86(2):174-179.

16. Fu H, Gu ZT, Fang WT, et al. Long-term survival after surgical treatment of thymic carcinoma: a retrospective analysis from the Chinese alliance for research of thymoma database. Ann Surg Oncol. 2016; 23(2):619-625.

17. Li W, Miao Z, Liu X, et al. Thymic carcinoma patients with myasthenia gravis exhibit better prognoses. Int J Clin Oncol. 2016;21(1): $75-80$

18. Koga K, Matsuno Y, Noguchi M, et al. A review of 79 thymomas: modification of staging system and reappraisal of conventional division into invasive and non-invasive thymoma. Pathol Int. 1994;44(5) 359-367.

19. Travis WD, Brambilla E, Müller-Hermelink HK, Harris CC, editors. WHO Classification of Tumours. Pathology \& Genetics of Tumours of the Lung, Pleura, Thymus and Heart. IARC Press: Lyon; 2004: 145-147.

20. Robinson JT, Thorvaldsdóttir H, Winckler W, et al. Integrative Genomics Viewer. Nature Biotechnology. 2011;29: 24-26.

21. Thorvaldsdóttir H, Robinson JT, Mesirov JP. Integrative Genomics Viewer (IGV): high-performance genomics data visualization and exploration. Briefings in Bioinformatics. 2013;14:178-192. 
22. Scheffler M, Bos M, Gardizi M, et al. PIK3CA mutations in nonsmall cell lung cancer (NSCLC): genetic heterogeneity, prognostic impact and incidence of prior malignancies. Oncotarget. 2015;6(2): 1315-1326.

23. Janku F, Wheler JJ, Westin SN, et al. PI3K/AKT/mTOR inhibitors in patients with breast and gynecologic malignancies harboring PIK3CA mutations. J Clin Oncol. 2012;30(8):777-782.

24. Wheler J, Hong D, Swisher SG, et al. Thymoma patients treated in a phase I clinic at MD Anderson Cancer Center: responses to mTOR inhibitors and molecular analyses. Oncotarget. 2013;4(6):890-898.

25. Kurup A, Burun M, Dropcho S, Pao W, Loehrer PJ. Phase II study of gefitinib treatment in advanced thymic malignancies. J Clin Oncol. 2005;23(16S):7068.
26. Suzuki E, Sasaki H, Kawano O, et al. Expression and mutation statuses of epidermal growth factor receptor in thymic epithelial tumors. Jpn J Clin Oncol. 2006;36(6):351-356.

27. Thomas A, Rajan A, Berman A, et al. Sunitinib in patients with chemotherapy-refractory thymoma and thymic carcinoma: an openlabel phase 2 trial. Lancet Oncol. 2015;16(2):177-186.

28. Welsh JS, Thurman SA, Howard SP. Thymoma and multiple malignancies: a case of five synchronous neoplasms and literature review. Clin Med Res. 2003;1(3):227-232.

29. Yen YT, Lai WW, Wu MH, Lin MY, Hsu IL, Tseng YL. Thymic neuroendocrine carcinoma and thymoma are both associated with increased risk of extrathymic malignancy: a 20-year review of a single institution. Ann Thorac Surg. 2011;91(1):219-225.
OncoTargets and Therapy

\section{Publish your work in this journal}

OncoTargets and Therapy is an international, peer-reviewed, open access journal focusing on the pathological basis of all cancers, potential targets for therapy and treatment protocols employed to improve the management of cancer patients. The journal also focuses on the impact of management programs and new therapeutic agents and protocols on

\section{Dovepress}

patient perspectives such as quality of life, adherence and satisfaction. The manuscript management system is completely online and includes a very quick and fair peer-review system, which is all easy to use. Visit http://www.dovepress.com/testimonials.php to read real quotes from published authors. 